\title{
Multi-Ensayos
}

Vol. 4, $\mathrm{N}^{\circ} 7$

ISSN: 2412-3285

https://multiensayos.unan.edu.ni

DOI: https://doi.org/10.5377/multiensayos.v4i7.9488

\section{Herramientas económicas para la toma de decisiones empresariales desde la asignatura de Economía Empresarial}

\section{Tools for Decision-making for Business Economics Students}

\author{
Samaria Ilú Alonso Valenzuela ${ }^{1}$
}

Recibido: 02 de marzo de 2018, Aceptado: 27 de septiembre de 2018

\section{RESUMEN}

En el presente ensayo, se abordan las herramientas económicas que se pueden utilizar para la toma de decisiones empresariales. La economía es la ciencia que estudia cómo se administran los escasos recursos, esta ciencia puede ser aplicada para el análisis macroeconómico y microeconómico, esto último relacionado a la dinámica económica de los agentes económicos en el mercado, estos fundamentalmente son las empresas y los consumidores. Para la toma de decisiones empresariales existen un sinnúmero de herramientas económicas que pueden ser utilizados por los empresarios para la formulación de su política de negocios, tales como análisis de oferta y demanda, análisis marginal para la determinación del punto óptimo de producción y maximización de beneficios, determinación de tipos de mercado dependiente de los números de compradores y vendedores, número de empresas participantes en el mercado, barreras de entrada y salida a los mercados, entre otros.

Palabras claves: herramientas económicas; toma de decisiones empresariales; economía.

\begin{abstract}
This essay describes different tools used for decision-making. Economics is the science that studies strategies to manage scarce resources. The principles of this science are useful in macroeconomic and microeconomic analysis, the latter related to the economic dynamics of economic agents in the market, these are mainly companies and consumers. For business decision making, there are countless tools that can be used by businessmen to plan their business policy, for example, the supply and demand analysis, marginal analysis to determine the optimum point of production and maximize profits, determination of market types depending on the numbers of buyers and sellers, the number of companies taking part and obstacles to enter and exit markets, among others.
\end{abstract}

Keywords: economic tools; business decision making; economy.

1 Docente del Departamento de Ciencias Económicas y Administrativas, UNAN-Managua/FAREM-Estelí. Correo electrónico: sama_ alvalen@yahoo.es

Copyright (c) 2018 Revista Multi-Ensayos. 


\section{INTRODUCCIÓN}

En la realidad empresarial, la mayoría de las empresas en Nicaragua, en especial las Micro, Pequeñas y Medianas Empresas (MIPYMES), no hacen uso de herramientas económicas que les permita la toma de decisiones efectivas y eficientes para desarrollarse en el mercado local mucho menos tener un proceso de internacionalización que les admita desarrollar su mercado internacional.

En el campo laboral la demanda de economistas es baja, esto debido a que las empresas locales desconocen la utilidad de la teoría económica para sus negocios. Es por esta razón que el objetivo del presente ensayo, es dar conocer como las herramientas de análisis económico son de gran utilidad para la formulación de políticas de negocios y por ende para tomar decisiones asertivas en las empresas.

Es por lo antes expuesto, que por medio de la asignatura de economía empresarial incorporada en la currícula de la carrera de Economía se les proporciona a los estudiantes las herramientas básicas para el análisis de la realidad empresarial y su aplicación práctica en la gestión de los negocios.

La asignatura está compuesta por ocho unidades donde se analizan temáticas macroeconómicas como microeconómicas, en la última unidad los estudiantes tienen la oportunidad de plantear ideas de negocios que satisfagan alguna necesidad local.

Así mismo, la asignatura tiene como finalidad analizar la realidad económica de las empresas y dar respuesta a los problemas económicos de la misma.

\section{DESARROLLO}

La economía es la ciencia que estudia cómo se administran los recursos de una sociedad, ya que ésta se enfrenta a una problemática y es la escasez de los recursos; es por esta razón que las unidades económicas tienen que encontrar la manera más eficiente de gestionar sus recursos, esto es aplicado al ámbito empresarial.

La economía de empresa intenta por medio de herramientas económicas aplicadas a la gestión de los recursos, que las empresas posean la información eficaz y eficiente para la toma de decisiones empresariales.

La economía se divide en dos grandes ramas de estudios: La microeconomía y la macroeconomía. La microeconomía estudia el comportamiento de los agentes económicos en el mercado, tales como las empresas y las familias. La macroeconomía estudia las variables agregadas, tales como el nivel general de precios, la producción nacional, el empleo, los salarios, así como el entorno internacional. La empresa se ve afectada por el entorno. Macroeconómicamente se ve afectada por las variables económicas tales como la inflación, los salarios, el mercado laboral, los impuestos, etc. Según el Banco Central de Nicaragua (Banco Central de Nicaragua (BCN) , 2015), la utilidad de las estadísticas económicas para las decisiones empresariales, está en la posibilidad de realizar estudios de nuevos mercados, o nuevas inversiones, análisis de entorno económico (inventario, precios, contratación de personal, etc.), realización de análisis técnico de ventas versus crecimiento económico, conocer la participación de la empresa en el sector y el destino 
de los productos que se producen, nos proporciona información de apoyo en el diseño de plan de ventas, en planeación de producción e inventarios, fijar metas y objetivos de corto y largo plazo; evaluación de como el comportamiento de las distintas variables macroeconómicas afectan la dinámica empresarial.

A nivel microeconómico, la economía proporciona a la empresa herramientas muy útiles para la asignación eficiente de los recursos, por ende, para la toma de decisiones asertivas; tales como análisis de demanda y oferta de mercado, asignación eficiente de niveles óptimos de producción, maximización de beneficios, entre otros.

Cada empresa o negocio tiene una meta. La teoría económica de la empresa asume que el principal objetivo de toda empresa es la de maximización de sus utilidades. Sin embargo, existen otras metas que la empresa puede conseguir relacionadas con la participación de mercado, el crecimiento de los ingresos, el margen de utilidad, el rendimiento sobre la inversión, la tecnología, la satisfacción al cliente y el valor para los accionistas (Keat \& Young, 2004).

Dependiendo de cuál sea la meta de la empresa, así serán las acciones a llevar a cabo; por ejemplo, si la meta es aumentar la participación en el mercado quizás la empresa decida reducir sus precios para incentivar la demanda, sin embargo, hay que tomar en cuenta el tipo de demanda de los productos o servicios o cuan sensibles son estos bienes antes las variaciones en los precios. Si la meta es la de proporcionar productos más avanzados tecnológicamente, decidirá invertir en investigación y desarrollo. Si la meta de la empresa es el aumento de los ingresos o aumento de las utilidades tendrá que analizar la asignación de los niveles de producción óptimos que maximicen los beneficios.

Dependiendo de la meta que se plantee cada negocio, se puede decir que la decisión óptima es aquella se acerca más a la empresa a su meta. Hay que diferenciar entre dos tipos de metas, aquellas que son de corto plazo y de largo plazo, esto va a estar determinado por las variaciones de factores fijos y variables (tales como mano de obra, capital fijo, etc.).

Una de las contribuciones más importantes que la teoría económica ha hecho a la toma de decisiones empresariales es la aplicación del análisis marginal. Éste se define como la cantidad que da cambio en una variable dependiente como resultado del cambio en una unidad en una variable independiente. (Keat \& Young, 2004). Por ejemplo, una empresa puede considerar variar los precios para obtener algún cambio en los ingresos o utilidades; sin embargo, este cambio dependerá del cambio en el número de unidades vendidas como resultados de la variación del precio.

Las cantidades demandadas de un bien que los consumidores deseen y puedan comprar las denominamos demanda de dicho bien (Mochón, 2005). Para analizar la variación que tendrá el número de unidades vendidas es fundamental analizar la demanda de los bienes; es decir, se tiene que determinar el tipo de demanda a la que se enfrentan los productos o servicios. Es fundamental para las empresas determinar el tipo de demanda al que se enfrentan los productos, ya que esto les afectará directamente los beneficios de la empresa; ya se elástica, inelástica o unitaria. 
La elasticidad se define como el cambio porcentual en la cantidad demandada ante variaciones porcentuales en el precio. Existe una relación directa entre la elasticidad, precio de la demanda y el ingreso recibido. Dado que la curva de demanda tiene pendiente negativa, una disminución del precio incrementaría la cantidad demandada del bien, y esto incrementará los ingresos. ¿Cuál de las dos tendencias es más fuerte? Esta es la pregunta clave que se debe responder con este tipo de análisis de elasticidad.

Dependiendo de cómo responde la cantidad demandada ante las variaciones en el precio es el resultado que obtendrá la empresa en sus ingresos. Si la demanda del bien es elástica un incremento del precio reducirá sus ingresos totales, ya que la disminución de la cantidad demandada será mayor el incremento del precio; sin embargo, cuando el bien tiene demanda elástica y decide reducir sus precios, el efecto en el ingreso será el alza. En el caso de la demanda inelástica, la respuesta es totalmente lo contrario, los ingresos suben cuando hay incrementos del precio y se reducen cuando hay disminuciones del precio.

La economía tiene vínculos muy fuertes con las funciones del negocio, por ejemplo con la mercadotecnia para el análisis de demanda, elasticidad precio, análisis de tipo de mercados; en las finanzas para la planeación del capital, análisis de punto de equilibrio, costo de oportunidad y valor económico agregado; en la contabilidad para el análisis de costo relevante, punto de equilibrio, costo incremental y costo de oportunidad; en la ciencia administrativa para el análisis de programación lineal, análisis de regresión lineal y pronósticos y en el área estratégica para la determinación de tipos de competencia y análisis de estructura-conducta-desempeño. (Keat \& Young, 2004). En la economía empresarial se retoman todos los análisis mencionados anteriormente de manera que transversa en las diferentes funciones de la empresa.

\section{CONCLUSIONES}

La economía de empresa es esencial para la formulación de las políticas de negocios. Haciendo uso de las herramientas económicas, las empresas pueden utilizarlas para la toma de decisiones asertivas en su negocio.

El análisis de la teoría económica aplicado al análisis microeconómico proporciona elementos para el desarrollo de habilidades empresariales en los estudiantes de economía, las cuales serán aplicadas a su campo laboral, debido a que localmente su campo laboral está más abierto al ámbito empresarial. A través de la economía de empresa los estudiantes pueden adquirir herramientas para el análisis económico de las empresas. Hacer uso de estas herramientas para la formulación de políticas de negocios, construcción de modelos económicos aplicados a los problemas en los negocios.

\section{BIBLIOGRAFÍA}

Banco Central de Nicaragua (BCN) . (2015, Agosto). Banco Central de Nicaragua. Retrieved Diciembre 15, 2016 from Banco Central de Nicaragua: https://goo.gl/81qcLT

Keat, P. G., \& Young, P. K. (2004). Economía de Empresa. México: Pearson Education. Mochón, F. (2005). Economía, teoría y política. Madrid: Mc Graw Hill. 